\title{
Pediatric Renal Transplantation: Comparative Study With Renal Transplantation in the Adult Population
}

\author{
B. Parada, A. Figueiredo, P. Nunes, C. Bastos, F. Macário, A. Roseiro, V. Dias, F. Rolo, and A. Mota
}

\begin{abstract}
Purpose. To retrospectively review our experience with pediatric renal transplantation and to compare the results with the adult population.

Patients and methods. Between January 1981 and August 2003, 74 renal transplants were performed in patients $\leq 18$ years at the time of the transplant-the pediatric group versus 1153 patients in the adult group. We analyzed various risk factors for actuarial kidney graft and patient survivals using the Kaplan-Meier method.

Results. Median ages were $13.8 \pm 3.5$ and $42.6 \pm 2.4$ years, respectively. There was no statistically significant difference in the human leukocyte antigen matching or immunosuppression. There was, however, a younger donor age and shorter ischemia time in the pediatric group. Overall, kidney transplant survival rates for patients $\leq 18$ years at $1,2,5$, and 10 years were $94.4 \%, 91.3 \%, 70.6 \%$, and $58.2 \%$, respectively, with no significant difference for patients older than $18(91.2 \%, 89.3 \%, 78.8 \%, 60.5 \%, P=.4325)$. There was a significantly decreased graft survival in the adult group at 10 years when the donor age was over 60 years and when the ischemia time was $\geq 20$ hours. The incidence of delayed graft function and the creatinine levels of functioning grafts did not differ between the two groups. During the follow-up, acute rejections were more frequent in the younger group. Patient survival in the pediatric group at $1,2,5$, and 10 years was $98.6 \%, 98.8 \%, 98.6 \%$, and $90.3 \%$, respectively, significantly lower in the adult group $(95.3 \%, 94.0 \%, 87.9 \%, 76.8 \%, P<.02)$.

Conclusions. Renal transplantation may be successfully performed in the pediatric patients with end-stage renal disease. Overall graft survival at 10 years did not differ significantly between the two groups. There is a higher incidence of acute rejections but longer patient survival in the pediatric population.
\end{abstract}

$\mathrm{R}$ ENAL TRANSPLANTATION is clearly the treatment of choice in children with end-stage renal disease (ESRD). ${ }^{1-3}$ However, many factors, including comorbidity and technical, immunological, and psychosocial aspects, make this population different from their adult counterparts. ${ }^{1}$ Although many reports show that graft survival in the pediatric population is inferior to that achieved in the adult population, ${ }^{4-8}$ the effect of age on graft survival has not been demonstrated by others. ${ }^{9,10}$ In this report, we describe our experience with renal transplantation in the pediatric compared with the adult group.

\section{PATIENTS AND METHODS}

Between January 1981 and August 2003, there were 1227 renal transplants. Seventy-four patients were under 18 years at the time of the transplant-the pediatric group-versus 1153 patients over 18 years-the adult group.
All clinical rejections (25\% increase in creatinine from baseline) were biopsy-proven and Banff-scored prior to initiation of therapy with high-dose steroids. Calculated creatinine clearance was assessed using the Cockroft-Gault formula.

The primary endpoints of this study were patient survival, graft survival, and the number of acute rejection episodes. Secondary endpoints included delayed graft function, serum creatinine, and calculated creatinine clearance.

Statistical analysis was performed using SPSS for Windows 10.0. Descriptive statistics are presented as mean values and standard deviations for the continuous variables and as frequencies and percentages for the categorical variables. Groups were compared

From the Department of Urology and Renal Transplantation, University Hospital of Coimbra, Coimbra, Portugal.

Address reprint requests to Belmiro Parada, Department of Urology and Renal Transplantation, University Hospital of Coimbra, 3000 Coimbra, Portugal. E-mail: parada.belmiro@netc.pt 0041-1345/05/\$-see front matter doi:10.1016/j.transproceed.2005.05.046 
Table 1. Characteristics of Patients at the Time of Renal Transplantation

\begin{tabular}{|c|c|c|c|}
\hline & $<18$ years $(n=174)$ & $>18$ years $(n=1153)$ & $P$ \\
\hline Recipient age & $13.8 \pm 3.5$ & $42.6 \pm 12.4$ & $<.01$ \\
\hline Gender (M/F) & $37 / 30(55.2 \% / 44.8 \%)$ & $728 / 350(67.5 / 32.5)$ & $<.01$ \\
\hline \multicolumn{4}{|l|}{ Causes of ESRD (\%) } \\
\hline Glomerulopathy & 28.4 & 20.4 & \\
\hline Tubulointersticial & 27.0 & 13.0 & \\
\hline Congenital & 16.2 & 14.2 & \\
\hline Metabolic (hypertension; diabetes) & 6.8 & 16.8 & \\
\hline Other/not determined & 21.6 & 35.6 & \\
\hline Dialysis (mo) & $15.1 \pm 18.6$ & $39.1 \pm 36.9$ & $<.01$ \\
\hline Donor age & $26.0 \pm 10.1$ & $32.3 \pm 14.9$ & $<.05$ \\
\hline Cold ischemia (h) & $17.9 \pm 8.1$ & $20.7 \pm 6.6$ & $<.05$ \\
\hline HLA matches & $2.3 \pm 1.0$ & $2.4 \pm 1.0$ & NS \\
\hline PRA (panel reactive antibodies) & $10.8 \pm 19.4$ & $7.56 \pm 14.3$ & $<.02$ \\
\hline Acute rejection & $0.49 \pm 0.50$ & $0.32 \pm 0.47$ & $<.01$ \\
\hline Acute tubular necrosis & $0.12 \pm 0.33$ & $0.17 \pm 0.38$ & $<.01$ \\
\hline Cadaveric/living donor (\% living donor) & $67 / 7(9.5 \%)$ & $1123 / 27(2.3 \%)$ & $<.01$ \\
\hline \multicolumn{4}{|l|}{ Causes of graft loss (\%) } \\
\hline Chronic dysfunction & 60 & 34.6 & \\
\hline Acute rejection & 8.0 & 5.0 & \\
\hline Technical complications & 8.0 & 4.4 & \\
\hline Disease relapse & 8.0 & 0.6 & \\
\hline Death with functioning graft & 8.0 & 39.4 & \\
\hline Primary nonfunctioning & 4.0 & 7.4 & \\
\hline Other & 4.0 & 8.6 & \\
\hline Follow-up (y) & $6.03 \pm 5.05$ & $5.97 \pm 4.53$ & NS \\
\hline
\end{tabular}

using Students $t$ test for continuous variables and categorical parameters were compared by $\chi^{2}$ testing. Graft and patient cumulative actuarial survival were calculated by Kaplan-Meier analysis and tested for differences with the Mantel-Cox log-rank test. Results were considered statistically significant if the $P$ value was less than or equal to .05 .

\section{RESULTS}

The patient characteristics at baseline are shown in Table 1. Median age in the pediatric and in the adult groups were $13.8 \pm 3.5$ and $42.6 \pm 2.4$ years, respectively. The causes of ESRD were obviously different (Table 1). There was no statistically significant difference in the human leukocyte antigen (HLA) matching $(2.3 \pm 0.95$ vs $2.4 \pm 1.0, P>.05)$ or immunosuppression. There was, however, a younger donor age $(26.0 \pm 10.1$ vs $32.3 \pm 14.9$ years, $P<.05)$ and shorter ischemia time $(17.9 \pm 8.1$ vs $20.7 \pm 6.6$ hours, $P<$ $.05)$ in the pediatric group. Overall, kidney transplant survival rates for patients $\leq 18$ years at $1,2,5$, and 10 years were $94.4 \%, 91.3 \%, 70.6 \%$, and $58.2 \%$, respectively, with no significant difference for patients older than 18 (91.2\%, $89.3 \%, 78.8 \%, 60.5 \%, P=.4325$; Fig $1 \mathrm{~A})$. When the observations were censored for patient death with a functioning graft, actuarial graft survivals at 1, 5, and 10 years were $95.0 \%, 75.3 \%$, and $72.6 \%$ in the pediatric patients, which were slightly lower than in the adult group (93.2\%, $87.4 \%$, and $79.6 \%$, respectively, $P<.05$; Fig $1 \mathrm{~B})$. Patients were further divided according to donor age $(\leq 18 ; 19$ to 59 ; $\geq 60$ years; Fig 2 ) and graft cold ischemia time $(<20 ; \geq 20$ hours). There was a significantly decreased graft survival in the adult group at 10 years when the donor age was over 60 years $(61.5 \%$ vs $37.7 \%, P<.05)$ and when the ischemia time was $\geq 20$ hours $(63.6 \%$ vs $58.4 \%, P<.05)$. Among the pediatric patients, there was no donor over 60 years and the ischemia time did not influence graft survival. During the follow-up $(6.03 \pm 5.05$ vs $5.96 \pm 4.53$ years, $P>.05)$, acute rejections were more frequent among the younger group $(0.49 \pm 0.50$ vs $0.32 \pm 0.47, P<.01)$. The occurrence of acute rejection episodes had a negative impact on long-term graft survival in the pediatric group (Fig 3). The incidence of delayed graft function was lower in the younger group $(0.12 \pm 0.33$ vs $0.17 \pm 0.38, P<.01)$ but the renal function (serum creatinine and creatinine clearance) did not differ between the two groups (Table 2). The causes of graft loss differed between the two groups (Table 1).

Patient survivals in the pediatric group at 1, 2, 5, and 10 years were $98.6 \%, 98.8 \%, 98.6 \%$, and $90.3 \%$, respectively, which were significantly greater than the adult group $(95.3 \%, 94.0 \%, 87.9 \%, 76.8 \%, P<.02$, Fig 4$)$.

\section{DISCUSSION}

Renal transplantation is the best replacement therapy for children with ESRD. ${ }^{1-3}$ Although several technical, immunological, metabolic, and psychosocial factors make them uniquely different from the adult population with a renal transplant, ${ }^{1}$ data about graft survival are conflicting. Although some groups did not find any difference in graft survival between the two age groups, ${ }^{9,10}$ most reports state that renal graft survival in the pediatric group is inferior, ${ }^{4-8}$ 

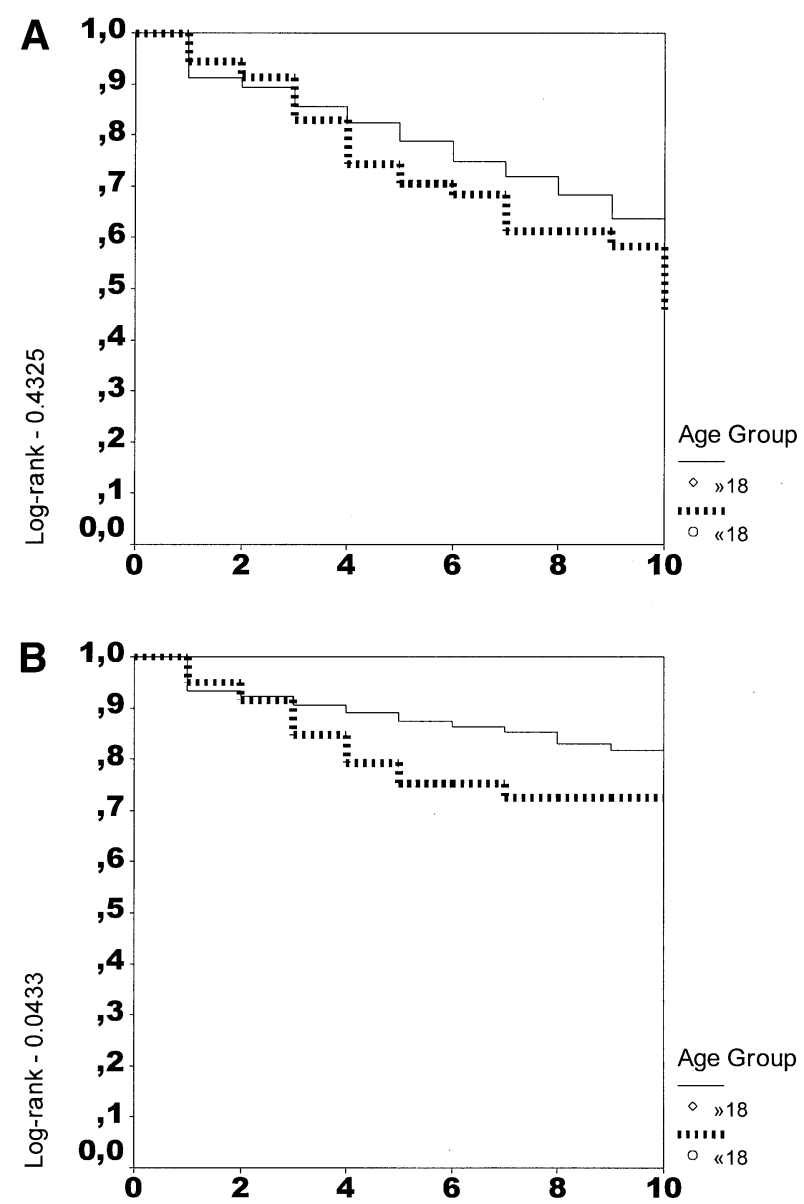

Fig 1. (A) Cumulative graft survival. (B) Cumulative graft survival (censored).

mainly due to a greater number of acute rejection episodes and technical complications, especially immediately in the posttransplant period. ${ }^{11-13}$ Noncompliance to the immunosuppressive medications is also a major factor in the late loss of renal allografts, especially among adolescents. ${ }^{7}$ Over the past decade, there has been a steady improvement in patient and graft outcomes in pediatric renal transplantation. ${ }^{2}$ Some of these improvements are due to more rigorous pretransplant evaluation, changing practice patterns, and better immunosuppression. ${ }^{14}$

In our series, the causes of ESRD differed between the two groups, as expected (Table 2), with congenital diseases being more frequent in the pediatric group and a greater prevalence of metabolic disorders (hypertension, diabetes) and undetermined etiology in the adult population.

Censored graft survival at 5 and 10 years were slightly higher in the adult group. The donor age was significantly lower in the children, because we did not use cadaveric kidneys from donors older than 60 for these patients. When analyzing the influence of donor age, we observed a shorter graft survival in the adults when the donor was older than 60 years. Increasing donor age has been shown to have a negative effect on graft survival. ${ }^{15-17}$ However, the impact of donor age is minimal when the mean donor age is 20 to 30 years but increases with higher and also with younger ages. ${ }^{9}$ Kidneys from cadaveric donors under 5 years provide the poorest graft survival in children. ${ }^{6,18}$ There was also a greater incidence of acute rejection episodes in children compared with the adults, with a negative impact on graft survival. This suggests a more vigorous immune response in children, as shown by a higher value of panel-reactive antibodies (PRAs). It also explains that chronic dysfunction is the leading cause of graft loss in this group.

The shorter ischemia time in the pediatric group can be explained by the higher frequency of living related donors, but it did not influence graft survival. On the contrary, in the adult population, ischemia time over 20 hours was a negative factor for long-term graft survival.

In conclusion, renal transplantation may be successfully performed in pediatric patients with ESRD. Overall graft survival and renal function at 10 years did not differ significantly between pediatric and adult populations. There was decreased graft survival among adult patients when the
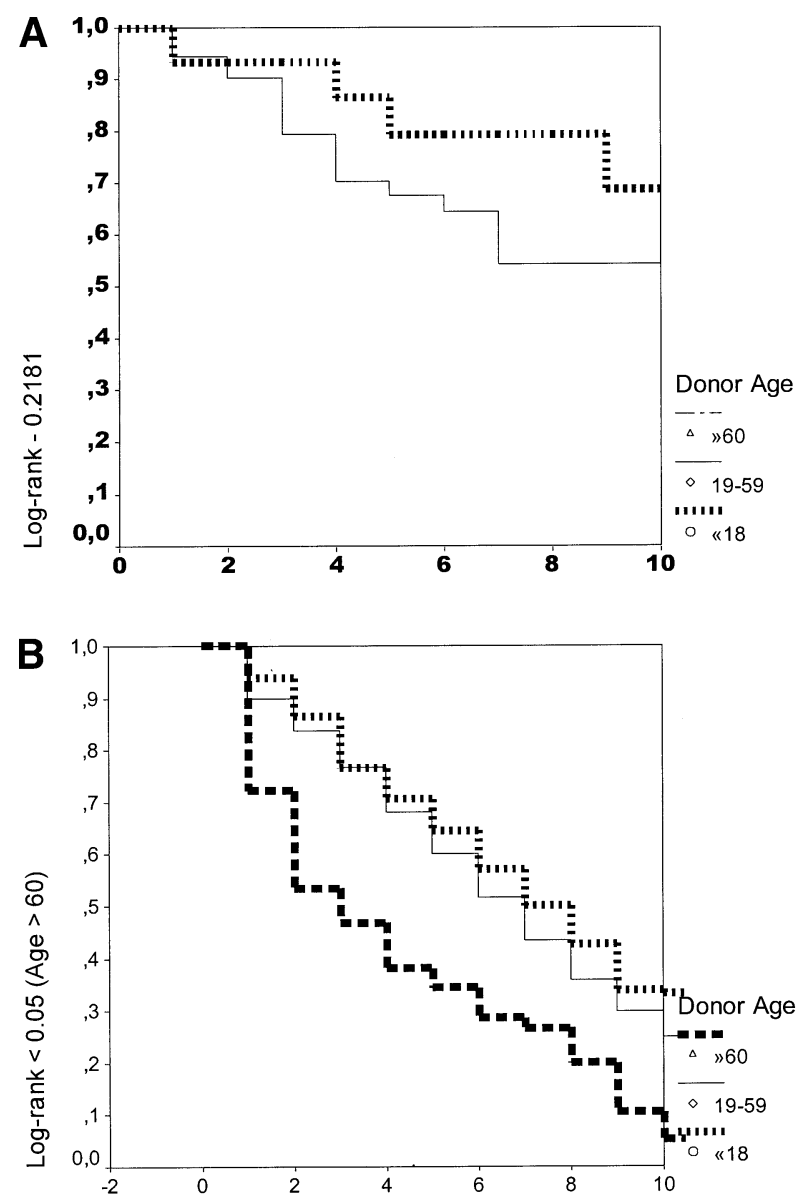

Fig 2. (A) Cumulative graft survival in the pediatric groupdonor age. (B) Cumulative graft survival in the adult groupdonor age. 
Table 2. Renal Function in the Two Groups

\begin{tabular}{|c|c|c|c|c|c|}
\hline \multirow[b]{2}{*}{ Renal function } & \multicolumn{2}{|c|}{$<18$ years } & \multicolumn{2}{|c|}{$>18$ years } & \multirow[b]{2}{*}{$P$} \\
\hline & Creatinine (mg/dL) & GFR $\left(\mathrm{mL} / \mathrm{min} / 1.73 \mathrm{~m}^{2}\right)$ & Creatinine $(\mathrm{mg} / \mathrm{dL})$ & $\operatorname{GFR}\left(\mathrm{mL} / \mathrm{min} / 1.73 \mathrm{~m}^{2}\right)$ & \\
\hline 1 month & $1.2 \pm 1.2$ & NA & $1.6 \pm 1.1$ & NA & NS \\
\hline 1 year & $1.2 \pm 0.5$ & $67.9 \pm 25.5$ & $1.4 \pm 0.5$ & $67.9 \pm 19.0$ & NS \\
\hline 5 years & $1.3 \pm 0.8$ & $66.2 \pm 16.2$ & $1.5 \pm 0.7$ & $70.8 \pm 22.7$ & NS \\
\hline 10 years & $1.5 \pm 0.8$ & $66.8 \pm 22.2$ & $1.5 \pm 1.0$ & $73.1 \pm 22.4$ & NS \\
\hline
\end{tabular}

GFR, glomerular filtration rate.

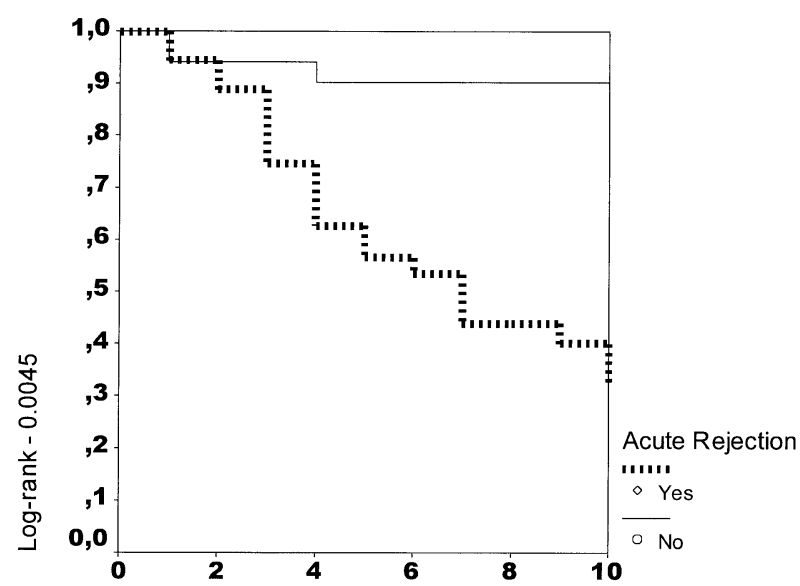

Fig 3. Cumulative pediatric graft survival-acute rejection.

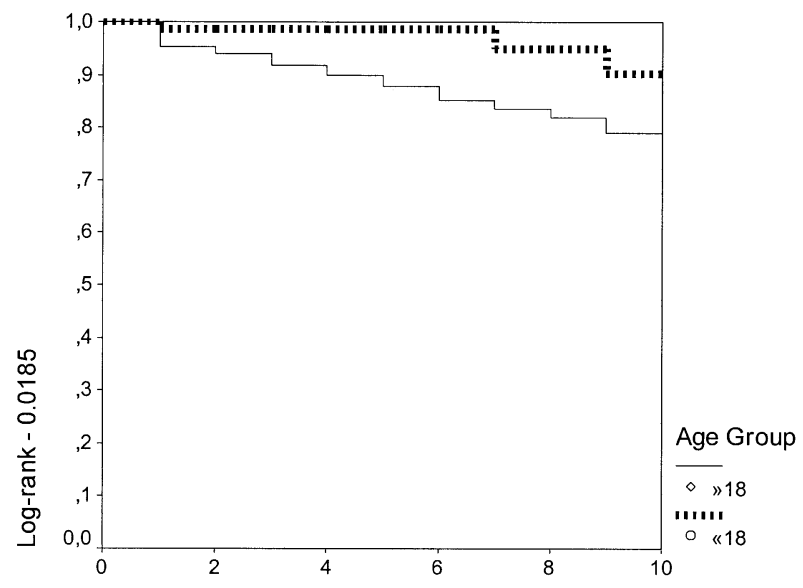

Fig 4. Cumulative patient survival.

donor age was over 60 years and cold ischemia time was more than 20 hours. There was a higher incidence of acute rejections and longer patient survival in the pediatric population, results that are in accordance with those in the literature. ${ }^{1,2,9,14,19,20}$ Renal transplantation in the pediatric group is an interdisciplinary task involving transplant surgeons, nephrologists, and intensive care physicians.

\section{REFERENCES}

1. Salvatierra O: Pediatric renal transplantation. Transplant Proc 31:1787, 1999
2. Smith JM, McDonald RA: Renal transplant outcomes in adolescents: a report of the North American Pediatric Renal Transplant Cooperative Study (NAPRTCS). Pediatr Transpl 6:493, 2002

3. European Best Practise Guidelines for Renal Transplantation: Section IV: long-term management of the transplant recipient. IV.11 Paediatrics (specific problems). Nephrol Dial Transplant 17(suppl 4):55, 2002

4. Berthoux FC, Jones EHP, Mehls O, et al: Transplantation report 1: renal transplantation in recipients aged 60 years or older at time of grafting. Nephrol Dial Transplant 11(suppl 1):37, 1996

5. Tesi RJ, Elkhammas EA, Davies EA, et al: Renal transplantation in older people. Lancet 343:461, 1994

6. Feld LG, Stablein D, Fivush B, et al: Renal transplantation in children from 1987-1996: the annual report of the NAPRTCS. Pediatr Transpl 1:146, 1997

7. Cecka JM, Gjertson DW, Terasaki PI: Pediatric renal transplantation: a review of the UNOS data. Pediatr Transpl 1:55, 1997

8. Salvatierra O, Tanney $\mathrm{D}$, Mak $\mathrm{R}$, et al: Pediatric renal transplantation and its challenges. Transpl Rev 11:51, 1997

9. Lufft V, Tusch G, Offner G, et al: Kidney transplantation in children: impact of young recipient age on graft survival. Nephrol Dial Transplant 18:2141, 2003

10. Lufft V, Kliem V, Tusch G, et al: Renal transplantation in older adults: is graft survival affected by age? A case control study. Transplantation 69:790, 2000

11. McDonald R, Ho PL, Tejani A: Rejection profile of recent pediatric renal transplant recipients compared with historical controls: a report of the NAPRTCS. Am J Transplant 1:55, 2001

12. Tejani A, Stablein EK, Sullivan EK, et al: The impact of donor source, recipient age, pre-operative immunotherapy and induction therapy on early and late acute rejections in children: a report of the NAPRTCS. Pediatr Transpl 2:318, 1998

13. Tejani A, Sullivan EK, Alexander S: Posttransplant deaths and factors that influence mortality rates in North American children. Transplantation 57:547, 1994

14. Seikaly M, Ho PL, Emmett L, et al: The 12th Annual Report of the North American Pediatric Renal Transplant Cooperative Study (NAPRTCS). Pediatr Transpl 5:215, 2001

15. Oberbauer Moreso F, Seron D, Gil-Vernet S, et al: Donor age and delayed graft function as predictors of renal allograft survival in rejection-free patients. Nephrol Dial Transplant 14:930, 1999

16. Morris PJ, Johnson RJ, Fuggle SV, et al: Analysis of factors that affect outcome of primary cadaveric renal transplantation in the UK. HLA task force of the UKTSSA. Lancet 354:1147, 1999

17. Terasaki PJ, Gjertson DW, Cecka JM, et al: Significance of the donor age effect on kidney transplants. Clin Transplant 11:366, 1997

18. Salvatierra O, Singh T, Shifrin R, et al: Successful transplantation of adult-sized kidneys into infants requires maintenance of high aortic blood flow. Transplantation 66:819, 1998

19. McDonald R, Donaldson L, Emmett L, et al: A decade of living donor transplantation in North American children: The 1998 Annual Report of the North American Pediatric Renal Transplant Cooperative Study (NAPRTCS). Pediatr Transpl 4:221, 2000

20. Pape L, Offner G, Ehrich JHH, et al: A single center clinical experience in intensive care management of 104 pediatric renal transplantations between 1998 and 2002. Pediatr Transplant 8:39, 2004 